\title{
FREE EXTERIOR POWERS
}

\author{
BY HARLEY FLANDERS ${ }^{1}$
}

Communicated by M. H. Protter, November 20, 1968

Our purpose is to announce several results concerning a unital module $M$ over a commutative ring $R$ with unity.

TheOREM 1. Let $M$ be a module over the ring $R$. Suppose for some $p$ that $\wedge^{p} M$ is free of rank one. Then

(i) $M$ is finitely generated, projective, and reflexive;

(ii) the module $M^{*}=\operatorname{Hom}(M, R)$ has the same properties, and $\wedge^{p} M^{*}$ is free of rank 1 ; and

(iii) for each $q, 0 \leqq q \leqq p$,

$$
\wedge^{e} M^{*} \approx\left(\wedge^{*} M\right)^{*} \approx \wedge^{p-a} M .
$$

CoRollary 1. If the module $M$ of Theorem 1 has a pure p-vector for a basis, then $M$ is free.

Corollary 2 (H. Osborn). If the ring $R$ in Theorem 1 is a local ring, then $M$ is free.

THEOREM 2. Let $M$ be a module over the ring $R$. Suppose for some $p$ that $\wedge^{p} M$ is cyclic. Then $\wedge^{p+1} M=0$.

Theorem 3. Let $M$ be a modille over the ring $R$. Suppose for some $p$ that $\wedge^{p} M$ is free of finite rank $q$. Then $\wedge^{p+q} M=0$.

A detailed paper will be submitted elsewhere.

Purdue University, Lafayette, Indiana 47907

${ }^{1}$ Research supported by NSF GP 6388. 